\title{
Memory and learning for a novel written style
}

\author{
JENNIFER ZERVAKIS and DAVID C. RUBIN \\ Duke University, Durham, North Carolina
}

\begin{abstract}
Subjects read and recalled a series of five short stories in one of four plot and style combinations. The stories were written in one of two styles that consisted of opposing clause orders (i.e., independentdependent vs. dependent-independent), tense forms (i.e., past vs. present), and descriptor forms (modifier modifier vs. modifier as a noun). The subjects incorporated both plot and style characteristics into their recalls. Other subjects, who, after five recalls, either generated a new story or listed the rules that had been followed by the stories read, included the marked forms of the characteristics they learned more often, except for tense. The subjects read and recalled four stories of the same plot and style and then read and recalled a fifth story of the same plot and style or of one of the other three plot/style combinations. Ability to switch style depended on both the characteristic and the markedness.
\end{abstract}

Story comprehension and memory research have traditionally focused on memory for the meaning of text, to the neglect of memory for the surface characteristics, or literary style. This focus was supported by a number of papers that had shown poor memory for surface form (Begg, 1971; Bransford, Barclay, \& Franks, 1972; Bransford \& Franks, 1971; D’Arcais, 1974; Sachs, 1967) but good memory for the propositional hierarchy of texts (Kintsch \& Van Dijk, 1978). The results of these studies, however, were due, at least in part, to researchers' having chosen texts with semantic organization but minimal stylistic organization. In contrast, oral traditions and literary texts have surface characteristics that are recurrent and interrelated (Rubin, 1995). The surface characteristics of these texts are not irrelevant but contain information regarding mood, symbolism, and artistic intent (Noice, 1991). Because surface information is important and must be learned in these cases, the appropriate question is not whether surface information is learned, but under what conditions and for what materials.

Memory for surface information is not typically tested in text studies, but it has been the focus of interest in a number of domains, such as prototype generalization (Franks \& Bransford, 1971; Posner \& Keele, 1968); artificial grammar learning (Dienes, Broadbent, \& Berry, 1991; Reber \& Lewis, 1977); and language acquisition, as in training neural nets to learn syntax (Plunkett \& Marchman, 1993). Here, the underlying similarities across examples are surface characteristics, and one finds that people do generalize across surface information. Other studies have demonstrated schema-like behavior for meaningless sentences (Katz \& Gruenwald, 1974) and paintings (Hartley

We thank Laura Kate Hennis for her assistance in testing subjects and scoring data. We thank Lynn Hasher, Amy Needham, Greg Lockhead, and Reiko Mazuka. We are grateful to Anjali Thapar and Denny LeCompte for their helpful comments on an earlier draft of this article. Correspondence concerning this article should be addressed to D. C. Rubin, Department of Psychology, Duke University, Durham, NC 27708-0086 (e-mail: rubin@psych.duke.edu).
\& Homa, 1981), in which surface information is generalized across examples.

Researchers who study literary style assert that stylistic text is different from normal text, and these differences may enable the surface information of stylistic texts to be learned and remembered. For example, stylistic texts have patterns and forms that are said to emphasize and highlight surface information. Stylistic texts are defined as a deviation from normal text or normal language use (Enkvist, 1973; Mukarovsky, 1970). This deviation typically refers to deviance in frequency, where stylistic texts contain a higher or lower frequency of certain word types, syntactic structures, or other markers than do normal texts. It should be noted that deviation in frequency is not entirely separate from deviation in quality, because sentences that are less common are more likely to be unusual and less grammatically preferred.

The difference in features in stylistic texts is often not just described as a difference in the frequency of features, but in how the features are systematically patterned in the text - what Freeman (1970) describes as a "recurrence or convergence of textual pattern" (p. 4) and Chapman (1973) calls pattern, the "selection and arrangement of items that contribute to the total effect" (p. 14). Rubin and Wallace (1989) state that the type of patterning found in rhythmic pieces may be especially effective for recall, because rhythm, alliteration, and meaning contribute different constraints that may more effectively specify a word than can a single constraint, such as meaning.

Another quality of style is that it is optional; it is a choice over other possibilities that are not restricted by grammatical or semantic constraints (Enkvist, 1973). This claim is reasonable for the abstract propositional meaning of a sentence, but it probably cannot be maintained for all levels of meaning. For instance, although a passive and an active can be represented by the same proposition, they differ in topicality, and topicality may be constrained by a number of factors, including focality (Olsen \& Filby, 1972), prototypicality (Kelly, Bock, \& Keil, 1986), and animacy (Bock, Loebell, \& Morey, 1992). These biases 
should be considered when examining memory for the form of sentences, so that styles are selected that are not determined by semantic or other constraints.

In psycholinguistics, when there is an optional choice between two forms, one form is considered marked and the other form unmarked. The unmarked optional form is the preferred form in both production and recall, and the marked form is the less preferred and less natural form. These preferences stem from a variety of reasons, but, suffice it to say, markedness can be used as a measure of deviance in optional stylistic forms.

It must be noted that, although this paper discusses syntactic and semantic information separately, these two types of information necessarily heavily interact whenever a sentence is comprehended or produced. This paper takes an extreme approach in examining surface characteristics that are not semantically determined. This is not to say that semantic information plays no role in style production. However, only by studying stylistic features that consist of optional forms can one have a strict test of demonstrating style learning independent of meaning differences.

The processing of stylistic text differs from that of nonstylistic text. The task demand for literary or artistic material may differ from text that is read for meaning. As comprehension is typically tested in story-reading tasks, memory for meaning is the assumed task when none is stated. However, studies have shown that memory for surface information improves when subjects are given processing tasks that increase attention for surface form, such as for rhyme (Morris, Bransford, \& Franks, 1977) or grammaticality (Graesser \& Mandler, 1975). Changes in task demand may also be prompted by the material itself. Rubin (1977) found that subjects' recall of "The Preamble" and Hamlet's soliloquy consisted essentially of correct words or omissions. A similar pattern was found in Hyman and Rubin's (1990) cued recall of Beatles' lyrics. As the subjects were not given verbatim instructions, Hyman and Rubin interpret the results as subjects' inferring the intended task as verbatim recall because of the particular characteristics of the material.

Differences in the stylistic text's material itself may induce different processing. McDaniel (1981) had subjects read sentences that varied in complexity while performing a comprehension-monitoring task. Memory for meaning was equivalent across sentences, but memory for form was better for the more complex sentences. A similar finding was found in the stylistic domain of paintings, where the subjects attended to different aspects of realist and abstract paintings. J. A. Schmidt, McLaughlin, and Leighten (1989) collected verbal protocols from novice and expert subjects on a set of realist and a set of abstract paintings. Because attention both to formal elements (lines, color relationships, shape) and to subject matter is needed to understand the picture, novices more closely resembled experts when analyzing abstract paintings than they did when analyzing realist paintings. The less accessible subject matter in the abstract paintings prompted subjects to attend to more formal elements and not just to subject matter. The authors suggest that the stylistic differences in abstract paintings increase attentive activity and slow comprehension, by making the familiar unfamiliar.

Miall and Kuiken (1994) found that text with more foregrounding (i.e., stylistic features) is processed differently than text with less foregrounding. They divided three literary stories into segments, and independent judges rated each segment for the amount of phonetic, syntactic, and semantic foregrounding. Novices, who were college students enrolled in an introductory psychology course, and experts, who were college students enrolled in senior literary courses, read and rated a story. For both novices and experts, the amount of foregrounding per segment was reliably associated with longer reading times and higher ratings of strikingness and affect. Patterning in stylistic text slows processing and may allow stylistic characteristics to be noticed and learned explicitly.

Stylistic experts appear to argue that the differences inherent in stylistic text are noticed and, perhaps, learned explicitly: Stylistic forms induce material-appropriate processing of surface form, because they are more unusual or difficult than normal text. However, there is also evidence to argue for the implicit learning of style. Implicit learning is said to occur when a person learns to respond to some criteria in an appropriate or rule-like manner, without the ability to verbalize the rules of that behavior. Historically, style has been described as best learned through imitation, and not through the learning of explicit rules (Aristotle, trans. 1976; Gorrell \& Laird, 1972, p. 428; Isocrates, trans. 1934). Lord (1960) studied Yugoslavian folk singers who were able to compose novel epic songs containing a large number of regularities but could not state these rules (e.g., they were not able to state what a line was).

Implicit learning is a concept under considerable dispute, with researchers disagreeing on measures to distinguish implicit learning and even whether implicit learning as a concept should be discarded (see Dienes \& Berry, 1997; Shanks \& St. John, 1994; Whittlesea \& Dorken, 1997, for discussions). The terms implicit and explicit are used in this paper in an inclusive sense to refer to a difference between verbalizable and performance-based knowledge. Under this usage, first-language acquisition can be said to be implicit, because people have a rich set of rules for use in production and judgements, yet have little verbalizable knowledge of those rules. The studies described in the paper do not apply strict tests of implicit learning - in part, because there is dispute over whether there are any available measures that can fulfill either sensitivity and information criteria (Shanks \& St. John, 1994) or both the exhaustiveness and the exclusiveness assumptions (Reingold \& Merikle, 1988). Rather, we would like to apply procedures and findings from the implicit-learning field to a more real-world domain. The 
question is not whether style learning is explicit or implicit. Rather, the issue is to determine the conditions and materials that seem to favor each kind of learning.

Previous studies that demonstrated learning for surface form used material that contained little or no semantic organization, such as prototype studies. These studies were important because they demonstrated generalization independent of the abstraction of semantic ideas. Ideally, style learning should be studied with texts that contain both stylistic and semantic organization. There are only a handful of psychological studies that fulfill this criterion. The first and most famous study is Bartlett's (1932) "War of the Ghosts." He found that subjects noticed the style but had poor productive control over it; they recalled the story with a more standardized style so that original stylistic differences were lost. However, the subjects read only one story of a style and thus had little opportunity to develop a mastery of that style. Bartlett himself states that, although reproduction was poor, subjects may react and even remember a narrative "largely because of its formal character" (Bartlett, 1932, p. 81).

Brewer and Hay (1984) looked at recalls of texts that were a mix of standard style and a particular genre. They also found evidence of reconstruction in which subjects retained the meaning of the phrases $51 \%$ of the time and the surface form only $16 \%$ of the time. Brewer and Hay also found evidence for memory for a schema, in this case a schema for a previously learned style. It is difficult in this study to separate the subjects' memory for semantic information and for surface information, because the subjects read passages in which the content and the style were confounded--a selection from the Bible was used as an example of biblical style. This study examines memory for previously learned genres; it does not address how such schemas of a style are initially learned.

Rubin, Wallace, and Houston (1993) used an expertise approach to memory for stylistic material, in which learning occurs over multiple sessions with related, but not identical, material. Novices learned five ballads over five sessions. For each ballad, they listened, recalled it, and then listened to it four more times before a second recall. After the fifth session, they completed a number of tasks that tested their knowledge of the ballad form. The tasks were generating a new ballad, listing rules to which the ballad conformed, completing a cloze task of partially incomplete ballad lines, and a forced choice of ballad lines that were changed by one or more constraints. In contrast to previous studies, novices did show learning of the multiple constraints of style. The composed ballads contained $62 \%$ of the ballad's possible constraints, whereas the rules list contained only $27 \%$. This study demonstrated style learning with naturalistic material, using a variety of measures. However, the choice of stimuli necessitated a loss in experimental control. The regularities and constraints found in the ballads were often codependent, so that learning for the various aspects of the text could not be manipulated and examined separately.

\section{EXPERIMENT 1}

Other studies have examined memory for natural, established stylistic forms (Brewer \& Hay, 1984; Rubin et al., 1993); the purpose of our first experiment was to determine whether the subjects would be able to learn a highly controlled, novel, arbitrary style. In this way, learning could not be attributed to matching to an already known style. The styles used were complex, in that the two styles were constructed to vary in tense, descriptor type, and clause order. Because each style used one of two opposing, alternative forms, it was not as critical to control for initial baseline levels of learning. The subjects were given a large and predictable amount of experience with the style, because every sentence of a particular style was of the same form. In addition, unlike most studies that look at learning for surface organization, the materials contained semantic organization as well, thus approximating the more natural conditions in which style is learned.

The experiments used a learning procedure common among the prototype-learning and expertise-learning fields but uncommon in text memory studies: generalization across successive examples (but see Thorndyke, 1977). A subject learns the style over a series of five stories in which the particular words, characters, and settings change, but the underlying style and plot does not. In this way, learning of the plot and the style can be examined separately from verbatim memory.

\section{Method}

Subjects. Twenty-one Duke undergraduates participated for partial fulfillment of a course requirement, with 10 subjects in the Style 1 group, and 11 in the Style 2 group.

Materials and Design. Two different plots and two opposing styles were constructed. Six short stories were written in each of two plot styles: the "Switch", in which a protagonist avoids trouble by switching identity, and "Just Desserts," in which a protagonist is harmed by his or her own plan for self-gain. Some plots were taken from Young (1961). For each plot type, the action of each of the 10 sentences in the story was specified in general terms. Each of these 12 stories was then written in two opposing styles containing three characteristics. The three style characteristics were chosen because (1) they changed surface structure, (2) they had little effect on the meaning of the story, and (3) there were at least two optional forms available. In this way, any style learning could be ascribed to memory for the form, rather than to differences in meaning. An effort was made to balance the more and less natural (unmarked vs. marked) forms across the two styles. The three style characteristics were clause order, descriptor type, and tense. Dependent-independent clause order is considered less natural than independent-dependent clause order in English, because English is a right-branching language that typically expresses the main clause first, with modifying clauses and phrases added later. The phrase descriptors were subjectively considered more unnatural. The present tense is considered marked, as compared with the past tense.

Style 1 consisted of sentences of the form: After she sneaks into the kitchen, Lucy, a child, steals a handful of picture perfect cookies. The sentences have a dependent-independent clause order, double descriptor ( picture perfect cookies) and present tense. Style 2 consisted of sentences of the form: Lucy, a child, stole a handful of pretty as a picture cookies after she sneaked into the kitchen. They 
have an independent-dependent clause order, phrase descriptor (pretty as a picture cookies), and past tense. See the Appendix for an example of stories written in the four plot/style combinations.

Each story contained 10 complex sentences. Because a natural style would not contain sentences entirely of the same syntactic form, these styles are necessarily somewhat artificial. However, constructing stories in which each sentence is of the same style gives the subjects the most experience with the style. Other studies have suggested that frequency of syntactic information may play a role in whether this information is used (Goodman, McClelland, \& Gibbs, 1981), and we did not want this to be a consideration.

Procedure. The between-subjects variables were plot and style, where plot and style were crossed so that the subjects learned one out of four possible plot-style combinations. The subjects were given five numbered booklets, each containing a cover sheet, a story, a distractor task page, and blank lined paper. For each read/recall trial, the subjects were given $2 \mathrm{~min}$ to read the story, $30 \mathrm{sec}$ to perform the distractor tasks, and 5 min to recall. Each distractor task was a series of number or letter puzzles in which the next item that followed in the series was to be filled in. The order of the stories were randomized, with the restriction that, across subjects, each story was seen in each position (1-5) the same number of times. The subjects were given the following instructions:

\begin{abstract}
In this study you will be required to do two main tasks. One of these tasks is completing a series of letter or number puzzles. To give you some practice, please complete this form (practice sheet for the distractor task is handed out). When doing the puzzles in the experiment, please try to complete as many as possible in the allotted time and do them in the order given to you. The other task is that you will read a series of five short stories. You will be given time to read each story about two times at your normal reading speed. After a short delay I will ask you to recall them. When you recall them, please recall them verbatim, or close to the original as possible. If you can't remember the stories exactly, please guess and do the best you can. Also, I will indicate when to open the booklets and also when to turn the pages. Once you have turned a page, please do not turn back to previous pages.
\end{abstract}

After the fifth recall, the subjects performed a similarityranking task, using sentences from a sixth, unread story. The subjects ranked nine sets of four sentences. The first sentence was not included because it was the same for all plots, a description of setting. They were instructed to rank each sentence in the set with a rank of 1 (most similar to the story sentences) to 4 (least similar). Each set of sentences consisted of the four combinations of plot and style, so that, for any particular subject, one sentence would have the same plot and style as those read, and the other three would be foils, differing in plot, style, or both.

\section{Results}

Because each story has 10 sentences that have three separable style characteristics, recalls of each story can be scored on a 10-point scale for each attribute. Similarly, each sentence has plot components that can be scored. Therefore, separate analyses of variance (ANOVAs) were performed for each dependent measure (clause order, descriptor type, tense, and plot).

For the descriptor scoring, a construction of the form "adjective or adverb as a noun" describing a noun was scored as a phrase descriptor (e.g., "gray as grit day" or "sunset as red as watermelon"). A construction of the form of "adjective adjective" or "adverb adjective" describing a noun was scored as a double descriptor. These con- structions did not need to use the same words as the original story, as form rather than meaning was scored.

For clause scoring, the sentences were first scored by the number of clauses they contained. To be scored as a clause, the construction needed to have both a stated subject and stated verb: "while he ran" was scored as a clause, "while running" was not. Although this does not conform exactly to what is linguistically considered to be a clause, all the clauses used in the story sentences followed this construction. Second, sentences of two or more clauses were scored as complex, compound, or compound/complex. Sentences were scored as complex if they had an independent and a dependent clause and did not have any independent compound clauses. An independent clause can stand alone as a sentence, and a dependent clause begins with a subordinate conjunction such as after, because, before, since, while, when. Compound clauses stand alone and are joined by coordinate conjunctions such as and, or, but. Third, the clause order for the complex sentences were scored by noting the order of the first two clauses.

The figures shown for the experiments list the number of style characteristics recalled, divided by the number of sentences recalled, to show the increase in the proportion of characteristics recalled per sentence. Plot scores are not normalized for the number of sentences recalled.

The first set of statistical analyses was not normalized, so that the dependent variables were the number of characteristics per recall. Plot analyses are reported later. The data were analyzed with a $2 \times 2 \times 5$ repeated measures ANOVA, where style read was a between-subjects variable with two levels (marked vs. unmarked), consistency of style produced was a within-subjects variable with two levels (consistent vs. inconsistent to style read), and serial position of the story was a within-subjects variable with five levels. Separate ANOVAs were done on the three dependent measures of clause order, descriptor type, and tense.

Table 1 lists all statistically significant $F$ s. For all three components of style, there was a main effect of consistency of style produced, so that there was a significant difference in the number of consistent versus inconsistent characteristics produced and a main effect of serial position. There was also a significant consistency-of-styleproduced $\times$ serial-position interaction, which appears to be due to the consistent characteristics increasing over stories, relative to the inconsistent characteristics. No other effects or interactions were significant. Not only were all style characteristics learned, style-read effects were nonsignificant, indicating that both forms were learned about equally.

The ANOVAs just reported could be affected by the raw amount of material remembered, as well as by a selective increase in the three style characteristics. To control for this, here and in all future analyses, the dependent measures were changed from the number of style characteristics produced to the number of style characteristics produced divided by the number of sentences recalled. 
Table 1

Statistically Significant $\boldsymbol{F}$ Tests for Style Characteristics in Experiment 1

\begin{tabular}{|c|c|c|c|c|c|c|c|}
\hline \multirow[b]{3}{*}{ Effect or Interaction } & \multirow[b]{3}{*}{$d f$} & \multicolumn{6}{|c|}{ Characteristics } \\
\hline & & \multicolumn{2}{|c|}{ Clause } & \multicolumn{2}{|c|}{ Descriptor } & \multicolumn{2}{|c|}{ Tense } \\
\hline & & $F$ & $M S_{\mathrm{e}}$ & $F$ & $\overline{M S_{\mathrm{e}}}$ & $F$ & $M S_{\mathrm{e}}$ \\
\hline Consistency of style produced & 1,19 & 31.99 & 4.94 & 114.88 & 7.67 & 838.99 & 3.44 \\
\hline Serial position & 4,76 & 6.50 & 0.96 & 4.74 & 93.29 & 6.65 & 0.57 \\
\hline Consistency $\times$ serial position & 4,76 & 2.55 & 1.57 & 4.22 & 0.98 & 6.98 & 4.50 \\
\hline
\end{tabular}

Figures 1-3 illustrate the number of consistent versus inconsistent characteristics produced per sentence for each style. The main effect of consistency of style produced remained for all three characteristics [clause order, $F(1,19)=$ 25.47, $M S_{\mathrm{e}}=0.09, p<.001$; descriptor, $F(1,19)=94.83$, $M S_{\mathrm{e}}=0.15, p<.001$; and tense, $F(1,19)=668.42, M S_{\mathrm{e}}=$ $0.06, p<.001]$. As can be seen in Figures 1, 2, and 3, the percentage of characteristics consistent with the style was significantly higher than that for nonconsistent characteristics. The effect of serial position remained only for clause $\left[F(4,76)=3.59, M S_{\mathrm{e}}=0.02, p<.05\right]$. For tense, the effect for serial position and the style-read $\times$ serialposition interaction could not be calculated because its $M S_{\mathrm{e}}$ was zero; if a sentence was recalled, its tense was correct. There was no longer a significant consistency-of-styleproduced $\times$ serial-position interaction for clause $[F(4,76)=$ $\left.1.98, M S_{\mathrm{e}}=0.03, p=.11\right]$ or for descriptors $[F(4,76)=$ $\left.0.96, M S_{\mathrm{e}}=0.02, p=.51\right]$. Only for clause order did the proportion of clause order sentences increase over stories. Thus, much of the increase in consistent characteristics over trials was due to a general increase in the amount of recall. Nonetheless, the main effect of consistency of style produced remained.

There are other ways to analyze the recall data, particularly for the clause order scoring. Figure 4 shows different possible measures, with the lines from top to bottom indicating the number of sentences per story, the number of sentences with more than one clause, the number of sentences that were complex sentences, and the number of complex sentences with correct clause order. Learning the correct clause order was not all or none, but dependent on learning a number of characteristics in parallel. In Figure 5, the lines from top to bottom indicate the number of sentences per story, the number of sentences with complex descriptors, and the number of correct complex descriptor forms. In contrast to clause order, if a complex descriptor was learned, the correct complex descriptor was learned. Tense is not shown, because the correct tense was used if the sentence was recalled, and so the tense results are the same as those shown by the top line in Figures 4 and 5.

For the plot, two independent scorers referred to both the original story and the list of plot actions. They were instructed to rank, from 1 (not at all close to the original) to 5 (extremely close to the original), how well the 10 original sentence meanings were recalled in the subject's recall, disregarding exact wording. The Pearson correlations coefficient score between the two plot scorers was
.79 , and the average of the two plot scores was used as the dependent measure. Figure 6 illustrates that the subjects learned the plot of the stories read. Plot recall scores were submitted to a $2 \times 5$ repeated measures ANOVA, where plot read was a between-subjects variable with two levels and serial position was a within-subjects variable with five levels. There was a significant effect for serial position $\left[F(4,76)=12.82, M S_{\mathrm{e}}=0.10, p<.001\right]$, but no significant effect for plot read $\left[F(1,19)=1.23, M S_{\mathrm{e}}=\right.$ $0.67, p=.28]$; both plots were equally well learned.

In the similarity-ranking test, the subjects ranked nine sets of four sentences from a sixth unread story; each sentence was ranked from 1 (most similar to the read stories) to 4 (least similar). Collapsing over the two plots and two styles, the sentences were ranked: same plot and style, 1.72; same style, 2.23; same plot, 2.74; and different plot and style, 3.24. The sentence ratings data were submitted to a $2 \times 2$ ANOVA, in which consistency of style read was a within-subjects variable with two levels and consistency of plot read was a within-subjects variable with two levels. Both consistency-of-style-read effects $[F(1,20)=27.45$, $\left.M S_{\mathrm{e}}=0.78, p<.001\right]$ and consistency-of-plot-read effects $\left[F(1,20)=12.16, M S_{\mathrm{e}}=0.44, p<.005\right]$ were significant, indicating that sentences that were consistent either with the style read or with the plot read were rated as more similar to the read stories than were sentences inconsistent

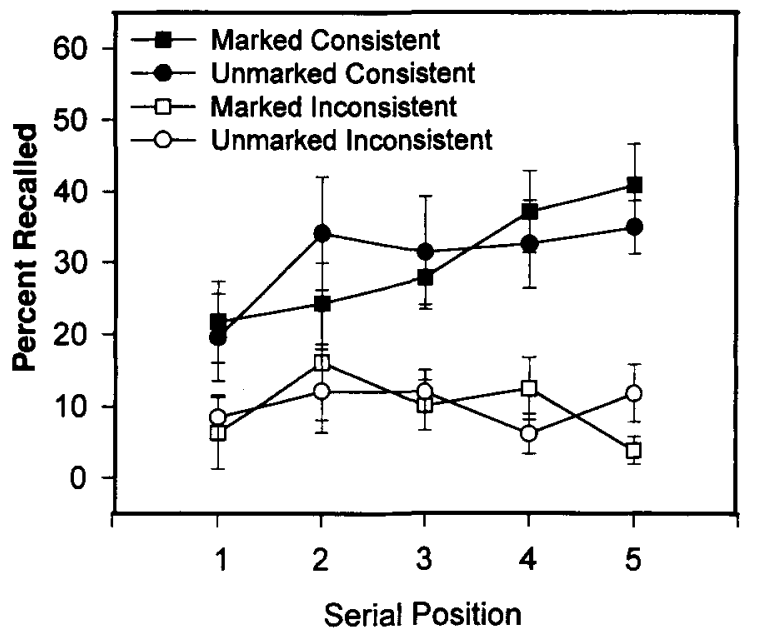

Figure 1. Percent of sentences recalled with consistent and inconsistent clause orders in Experiment 1, where marked is dependentindependent clause order and unmarked is independentdependent clause order. Error bars are standard errors. 


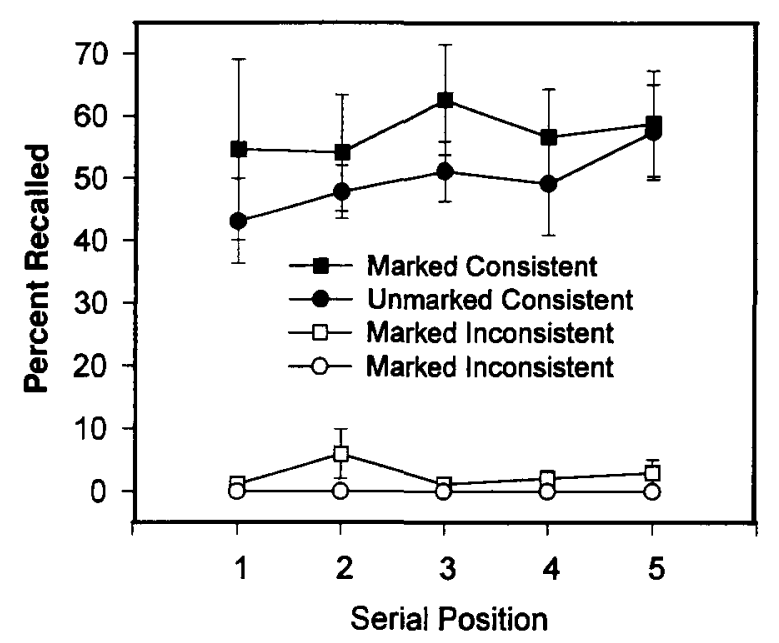

Figure 2. Percent of consistent or inconsistent descriptor types recalled in Experiment 1, where marked is phrase descriptors and unmarked is double descriptors. Error bars are standard errors.

with the style read or the plot read. There was no interaction of consistency of style read and consistency of plot read $\left[F(1,20)=0.0, M S_{\mathrm{e}}=0.08, p>.05\right]$. Thus, consistency of plot and style had additive effects on sentence rankings.

\section{Discussion}

The style characteristics were learned and incorporated into the recalls, as demonstrated by a significant consistency-of-style-produced effect for all characteristics. Although there was variability across recalls, both marked and unmarked forms were equally well learned by the fifth story. As can be seen in Figures 2, 3, and 6, the plot and the style characteristics tense and descriptor type were learned to a high degree from the first recall. Thus, when normalized by the number of sentences recalled, two of the three style characteristics showed no learning, being at a high level from the first story read. This rapid learning is similar to the Rubin et al. (1993) study, in which gist, rhyming words, and line structure were learned to a high degree by the end of the first session. Tense was learned especially well. The characteristic that did show learning over serial position when normalized was clause order, where proportion of correct clause orders continued to increase over stories.

\section{EXPERIMENT 2}

Rubin et al. (1993) found that subjects were able to produce stylistic regularities better than they were able to verbally describe them. In addition, there was no statistical relation between the number of times subjects used a rule and the number of times it was stated. This is similar to findings in the implicit-learning domain, where the ability to perform the stated task is dissociated from the ability to verbalize the methods used to perform the task.
The intent here was to replicate this finding with three additions. The first was the use of a novel style, in which learning to use or know the style could not be attributed to past familiarity with the style. The second was the use of stylistic materials in which the individual style characteristics and the plot were independent of each other. The third was a generate-story versus rules-listing manipulation that was a between-subjects variable, unlike the Rubin et al. study, because it was thought that the listrules and generate-story tasks would influence each other in the single-session design.

\section{Method}

Subjects. Forty-eight Duke undergraduates participated for partial fulfillment of a course requirement, with 24 in the generatestory group and 24 in the list-rules group.

Procedure. The subjects read and recalled five stories of the same plot and style. After the fifth recall, the subjects were placed in either the generate-story or list-rules group. The generate-story group was given a blank lined sheet with instructions similar to the ones used in the Rubin et al. (1993) study:

One way to test if you have learned the rules and structures of these stories is to have you generate a new one. Therefore, I would like you to try to make up a story that a critic would find difficult to pick out as yours if it were mixed among the set of five stories you learned.

The list-rules group was given a blank lined sheet with the following instructions:

You probably have noticed that the five stories you have read share many characteristics. Please record all the rules, generalizations, or properties that apply to the stories you read. For instance, if you read a series of poems and noticed that the first and second lines rhymed, you would state below "ends of first and second lines of stanzas rhyme."

Please include comments on both the content and structure.

\section{Results}

For the generate-story group, the new story was scored for style and plot characteristics, using the same methods as those used to score recalls in Experiment 1. The data were subjected to a $2 \times 2$ ANOVA, with style read a be-

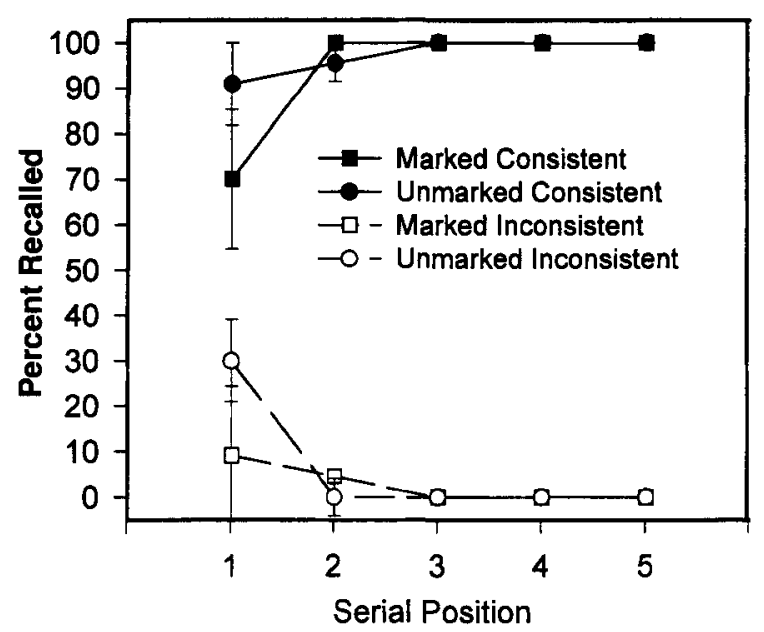

Figure 3. Percent of sentences recalled with consistent and inconsistent tense in Experiment 1, where marked is present tense and unmarked is past tense. Error bars are standard errors. 


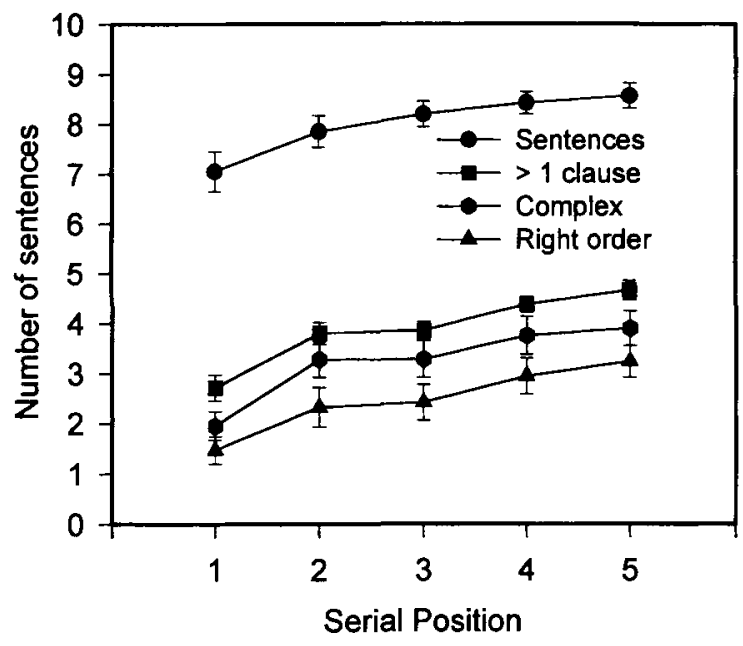

Figure 4. Total number of sentences recalled in Experiment 1 with more than one clause, complex connectives, and consistent clause order. Error bars are standard errors.

tween-subjects variable with two levels (marked vs. unmarked) and consistency of style produced a within-subjects variable with two levels (consistent vs. inconsistent). Separate ANOVAs were done on the three dependent measures of clause order, descriptor type, and tense. The number of style characteristics produced, divided by the number of sentences recalled, was the dependent variable and is shown in Table 2. Significant $F$ tests are shown in Table 3.

For the generate-story task, all style characteristics had significant consistency-of-style-produced effects (except for a marginal clause order), style-read effects, and style-read $\times$ consistency-of-style-produced interactions. Thus, the subjects were able to incorporate the style read in a new story. For descriptors and clause order, those subjects who learned the marked form generated a higher percentage of consistent style characteristics than those who learned the unmarked form (see Table 2). For tense, the opposite pattern was found: the subjects produced the unmarked past tense perfectly in the new story, but those who read the present tense were poorer at producing the correct tense in the new story.

The subjects for both plot types were able to generate stories with the appropriate plot. Both plots were about equally generated, with average plot scores of $3.14(S D=$ 0.72 ) for Switch and $3.00(S D=0.54)$ for Just Desserts (the Pearson correlations coefficient score between the two scorers was .61). As can be seen in Table 2, this indicates that the subjects incorporated about $62 \%$ of the total possible rules for the Switch and $60 \%$ of the rules for Just Desserts. The generated-story plot scores are comparable to the plot scores for the story recalls by the fifth recall.

In the list-rules group, the percentage of total possible rules verbalized for plot, clause order, descriptor type, and tense was scored. The 10 plot rules were the actions specified for the 10 sentences. There were three clause order rules, referring to the number of clauses, the conjunctions used, and the clause order. There were two descriptor rules, referring to the number of adjectives and the form of the description, and there was one tense rule. As can be seen in Table 2, for all style characteristics, the subjects were more likely to list rules for the marked style characteristics, although this difference was significant only for descriptors $[t(22)=3.95, p<.001$; clause, $t(22)=1.22$, $p>.05$; tense, $t(22)=1.20, p>.05$ ].

\section{Discussion}

Experiment 2 demonstrated that subjects, when asked to generate a new story of the same type, incorporated a higher percentage of the more unusual or marked style type in the generated story. For clause order, this was the dependent-independent clause order; for descriptors, the phrase descriptor. Tense was an exception: the subjects reverted to past tense in the generated story. The descriptor and clause order results contradict the common finding that sentence production is based on the more common form in the language. In most studies, recall follows normal production biases, so that the actual form of the sentence is replaced by more common forms - such as passive to active (D'Arcais, 1974; Miller, 1962) or dependent clause first to independent clause first (Jou \& Harris, 1990). Although this occurred for tense, the opposite was found for descriptor and clause order: the subjects produced the more distinctive style to a higher degree than they did the one that is common in the language as a whole.

Rather than demonstrating dissociations, the total percentage of rules verbalized for the list-rules group generally paralleled performance in the generate-story task, both in the percentage of total rules verbalized across style characteristics and in marked versus unmarked forms. As can be seen in Table 2, tense was an exception. The subjects showed a high rate of correct tense production in the story recalls of Experiment 1 and in the story generation of Experiment 2, but low tense rule listing in the list-rules task. In particular, past tense was generated

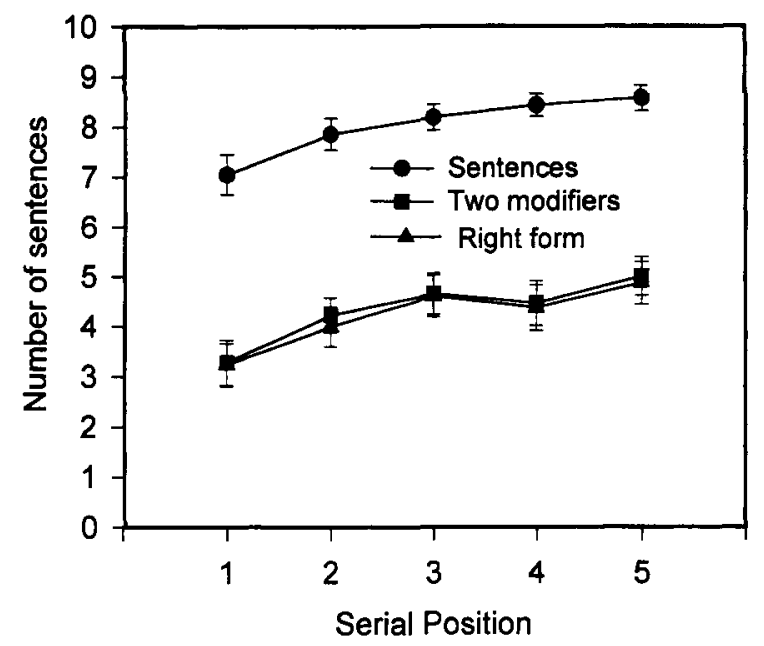

Figure 5. Total number of sentences recalled in Experiment 1 with complex descriptors and consistent descriptor types. Error bars are standard errors. 


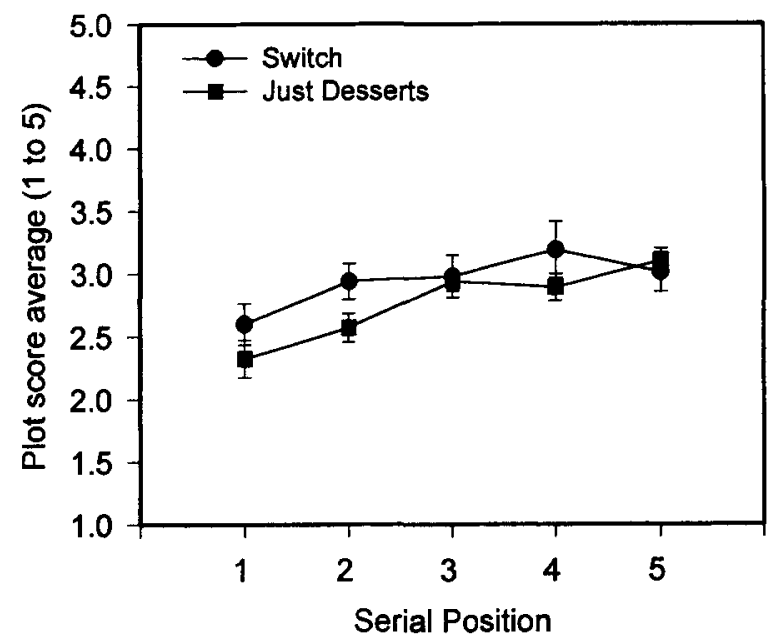

Figure 6. Average score for correct plot. Error bars are standard errors.

perfectly in the new story but was never mentioned as a rule.

It is presumed that the clause and descriptor findings are due to the marked characteristic being more noticeable. Other psychological studies have shown increased memory for distinctive material, presumably from increased attentive activity (S. R. Schmidt, 1991). The fact that the subjects appeared to attend to and produce the more unnatural forms of the style supports the view of style researchers who maintain that a characteristic must be distinctive from the norm to be considered part of a style.

Tense was the only style characteristic to show opposing patterns in list-rules and production performances. This is consistent with tense being learned implicitly. However, other researchers have noted that dissociations between measures do not constitute proof of implicit learning (Reingold \& Merikle, 1988; Shanks \& St. John, 1994); free report of rules may not be as sensitive or as exhaustive a measure of knowledge as performance.

\section{EXPERIMENT 3}

The third experiment was designed to determine whether learning a style would interfere with recalling a story of a different style, plot, or both. It was predicted that, if style is explicitly learned, switching the style in the fifth story should not be difficult; subjects would consciously note the change in rules and change their behavior appropriately. Moreover, the ability to switch forms should interact with the markedness of the original style. Switching from the unmarked to marked form should actually be easier, because the style would become more salient. In contrast, implicit-learning studies have shown a decrement in performance when an underlying rule is changed (Hayes \& Broadbent, 1988; Lewicki, Czyzewska, \& Hoffman, 1987). This is because, for implicitly learned preferences, the biases would have been built up from successive ex- perience and are not expected to be either verbalizable or particularly flexible.

\section{Method}

Subjects. Seventy-five Duke University undergraduates participated for partial completion of a course requirement, with 18,17, 21, and 19 subjects in Same Style 1, Same Style 2, Switch Style 1, and Switch Style 2 groups, respectively.

Procedure. Four groups of subjects read and recalled four stories of the same plot and style combination. For the fifth story, the subjects read a story of either the same plot and style, the opposite plot with the same style, the opposite style with the same plot, or the opposite plot and style.

\section{Results}

Initial learning. Descriptors, clause order, tense, and plot were scored as in Experiment 1. Stories 1, 4, and 5 were scored. The recalls from Story 1 and Story 4 were compared in order to replicate the learning of style characteristics found in Experiment 1. They were submitted to a $2 \times 2 \times 2 \times 2$ ANOVA, with style read a betweensubjects variable with two levels (marked vs. unmarked), consistency of style produced a within-subjects variable with two levels (consistent vs. inconsistent), serial position of the stories a within-subjects variable with two levels (1 vs. 4), and switch a between-subjects variable with two levels (style switches vs. style does not switch). Separate ANOVAs were done on the three dependent measures of clause order, descriptor type, and tense. The number of characteristics recalled, divided by the number of sentences, was used as the dependent variable.

Significant $F$ tests are shown in Table 4. For all three characteristics, there was a significant consistency-ofstyle-produced effect and a significant serial-position effect. For tense, style read, and serial position, effects could not be calculated, because $M S_{\mathrm{e}}=0$. For descriptor, there was a significant style-read effect. There were no other ef-

Table 2

Percentage of Style Characteristics Produced in Generate-Story Condition and Percent Total Rules Verbalized in List-Rules Condition

Style Produced

\begin{tabular}{lccc}
\hline & \multicolumn{2}{c}{ Style Produced } & \\
\cline { 2 - 3 } & Consistent & Inconsistent & Style Listed \\
\hline Style Characteristics & & & \\
$\quad$ Clause & & & \\
$\quad$ Marked & 30.6 & 9.8 & 11.1 \\
$\quad$ Unmarked & 10.2 & 10.8 & 2.8 \\
$\quad$ Descriptor & & & \\
$\quad$ Marked & 82.3 & 3.0 & 79.2 \\
$\quad$ Unmarked & 36.5 & 1.2 & 25.0 \\
$\quad$ Tense & & & \\
$\quad$ Marked & 58.3 & 41.7 & 8.3 \\
$\quad$ Unmarked & 100.0 & 0.0 & 0.0 \\
Plot Type & & & \\
$\quad$ Switch & 62.0 & & 42.5 \\
$\quad$ Desserts & 60.0 & & 43.3 \\
\hline
\end{tabular}

Note-Marked forms were dependent-independent clause order, phrase descriptor, and present tense. Subjects did not list rules for the nonread style. 
Table 3

Statistically Significant $\boldsymbol{F}$ Tests for Style Characteristics in Experiment 2

\begin{tabular}{|c|c|c|c|c|c|c|c|}
\hline \multirow[b]{3}{*}{ Effect or Interaction } & \multirow[b]{3}{*}{$d f$} & \multicolumn{5}{|c|}{ Characteristics } & \\
\hline & & \multicolumn{2}{|c|}{ Clause } & \multicolumn{2}{|c|}{ Descriptor } & \multicolumn{2}{|c|}{ Tense } \\
\hline & & $F$ & $M S_{\mathrm{e}}$ & $F$ & $M S_{\mathrm{e}}$ & $F$ & $M S_{\mathrm{e}}$ \\
\hline Consistency of style produced & 1,22 & 3.91 & 0.03 & 142.00 & 0.03 & 15.40 & 0.26 \\
\hline Style read & 1,22 & 7.16 & 0.02 & 19.33 & 0.04 & n.c. & n.c. \\
\hline Style read $\times$ consistency & 1,22 & 4.41 & 0.03 & 20.94 & 0.03 & 7.86 & 0.26 \\
\hline
\end{tabular}

Note-For clause, consistency of style produced, $p=.06$. For tense, style-read effect could not be calculated (n.c.), because $M S_{\mathrm{e}}=0$.

fects or higher order interactions, except for a consistencyof-style-produced $x$ serial-position interaction for clause and descriptor and a style-read $\times$ consistency-of-styleproduced interaction for tense.

The Story 1 and Story 4 recall results are similar to those of Experiment 1, in that the subjects learned the style. They differ from the results of Experiment 1 in that descriptors demonstrated significant serial-position and style-read effects; marked forms had a recall advantage. For tense, the opposite pattern was seen; the unmarked group learned tense perfectly. Tense demonstrated a styleread $X$ consistency-of-style-produced interaction, which appears to be due to the subjects producing a higher proportion of consistent tense sentences if they read the unmarked style.

Because of the differences between Experiments 1 and 3 , a post hoc analysis of Story 1 versus Story 4 data was performed in order to examine learning across experiments. Story 1 and Story 4 data from Experiment 2 were scored and combined with Story 1 and Story 4 recall data from Experiments 1 and 3. The three style characteristics were analyzed separately with a $2 \times 2 \times 2 \times 3$ ANOVA, with style read a between-subjects variable with two levels (marked vs. unmarked), consistency of style produced a within-subjects variable with two levels (consistent vs. inconsistent), serial position of the stories a within-subjects variable with two levels ( 1 vs. 4 ), and experiment a between-subjects variable with three levels (Experiment 1, 2, or 3).

Significant $F$ tests are shown in Table 5. For all style characteristics, there was a significant consistency-ofstyle-produced effect, a significant serial-position effect, and a significant consistency-of-style-produced $\times$ serialposition interaction. Subjects increased the proportion of consistent characteristics produced from serial position 1 to 4 . Regarding style-read effects, there was a significant style-read effect for descriptor but not for clause order. Although marked forms are more noticeable, they may be more difficult to produce, so that style-read effects are not expected to be found in all production tasks. Descriptor also showed a significant effect for experiment, indicating variability across experiments. It should be noted that serial-position effects for descriptor and tense and style-read effects for descriptor are reliably found only with a large power and are small, as compared with the very large consistency-of-style-produced effects found for all three characteristics.

No-switch condition. For recall results for Stories 4 and 5 , the groups for which style remained the same and the groups for which style switched in the fifth story were analyzed separately. For the no-switch group, the data from Story 4 and Story 5 were submitted to a $2 \times 2$ $\times 2$ ANOVA, with style read a between-subjects variable with two levels, consistency of style produced a withinsubjects variable with two levels, and serial position a within-subjects variable with two levels (4 vs. 5 ).

For the no-switch group, all three characteristics demonstrated a significant consistency-of-style-produced effect [clause, $F(1,34)=53.66, M S_{\mathrm{e}}=0.06, p<.001$; descriptor, $F(1,34)=233.56, M S_{\mathrm{e}}=0.05, p<.001$; and tense, $\left.F(1,34)=261.06, M S_{\mathrm{e}}=0.11, p<.001\right]$. There were no significant effects for style read or serial position. There were no significant interactions, with the exception, for tense, of a style-read $\times$ consistency-of-style-produced

Table 4

Statistically Significant $\boldsymbol{F}$ Tests for Style Characteristics in Experiment 3, Story 1 Versus Story 4

\begin{tabular}{|c|c|c|c|c|c|c|c|}
\hline \multirow[b]{3}{*}{ Effect or Interaction } & \multirow[b]{3}{*}{$d f$} & \multicolumn{6}{|c|}{ Characteristics } \\
\hline & & \multicolumn{2}{|c|}{ Clause } & \multicolumn{2}{|c|}{ Descriptor } & \multicolumn{2}{|c|}{ Tense } \\
\hline & & $F$ & $M S_{\mathrm{e}}$ & $F$ & $M S_{\mathrm{e}}$ & $F$ & $M S_{\mathrm{e}}$ \\
\hline Consistency of style produced & 1,71 & 136.48 & 0.04 & 526.83 & 0.04 & 181.38 & 0.24 \\
\hline Style read & 1,71 & n.s. & n.s. & 6.92 & 0.04 & n.c. & n.c. \\
\hline Serial position & 1,71 & 14.68 & 0.01 & 7.73 & 0.02 & n.c. & n.c. \\
\hline Consistency $\times$ serial position & 1,71 & 11.48 & 0.03 & 8.61 & 0.03 & n.s. & n.s. \\
\hline
\end{tabular}

Note-Style-read, switch, and serial-position effects for tense could not be calculated (n.c.) because $M S_{\mathrm{e}}=0$. For tense, style-read $\times$ consistency-of-style-produced interaction, $F(1,71)=$ $15.01, M S_{\mathrm{e}}=0.25, p<.001$. n.s. $=$ not significant. 
Table 5

Statistically Significant $\boldsymbol{F}$ Tests for Style Characteristics

in Experiments 1, 2, and 3 for Story 1 Versus Story 4

\begin{tabular}{|c|c|c|c|c|c|c|c|}
\hline \multirow[b]{3}{*}{ Effect or Interaction } & \multirow[b]{3}{*}{$d f$} & \multicolumn{6}{|c|}{ Characteristics } \\
\hline & & \multicolumn{2}{|c|}{ Clause } & \multicolumn{2}{|c|}{ Descriptor } & \multicolumn{2}{|c|}{ Tense } \\
\hline & & $F$ & $M S_{\mathrm{e}}$ & $F$ & $M S_{\mathrm{e}}$ & $F$ & $M S_{\mathrm{e}}$ \\
\hline Consistency of style produced & 1,138 & 139.40 & 0.04 & 663.75 & 0.05 & 273.59 & 0.25 \\
\hline Style read & 1,138 & n.s. & n.s. & 7.94 & 0.04 & n.c. & n.c. \\
\hline Experiment & 2,138 & n.s. & n.s. & 4.19 & 0.04 & n.c. & n.c. \\
\hline Serial position & 1,138 & 31.35 & 0.01 & 9.66 & 0.02 & 19.03 & 0.00 \\
\hline Consistency $\times$ serial position & 1,138 & 18.65 & 0.03 & 8.00 & 0.03 & 13.14 & 0.09 \\
\hline
\end{tabular}

Note-Style-read and experiment effects for tense could not be calculated (n.c.), because $M S_{\mathrm{e}}=0$. For tense, style-read $\times$ consistency interaction, $F(1,138)=8.75, M S_{\mathrm{e}}=0.25, p<$ .005 . n.s. $=$ not significant.

interaction $\left[F(1,34)=5.10, M S_{\mathrm{e}}=0.11, p<.05\right]$. The lack of serial-position and style-read effects replicate Experiment 1's findings of little additional learning from the fourth to the fifth story and of equal learning of marked and unmarked forms by the fifth recall. Having established a partial replication of Experiment 1, we turn to the interesting questions.

Switch-style condition. Figures 7,8 , and 9 illustrate the proportion of inconsistent and consistent characteristics recalled for the switch-style groups for Stories 1, 4, and 5. As can be seen in Figures 7, 8, and 9, subjects were generally able to switch style. Data from Story 4 and Story 5 were submitted to a $2 \times 2 \times 2$ ANOVA, with style read a between-subjects variable with two levels, consistency-of-style-produced a within-subjects variable with two levels, and serial position a within-subjects variable with two levels (4 vs. 5). Significant $F$ tests are shown in Table 6. All three characteristics demonstrated significant consistency-of-style-produced effects. There was an expected consistency-of-style-produced $\times$ serialposition interaction for all three characteristics (except for a marginal effect for descriptor), indicating that the overall proportion of consistent, as compared with inconsistent, characteristics decreased when style was switched. The expected style-read $\times$ consistency-ofstyle-produced $\times$ serial-position interaction was significant for descriptor and tense only. As can be seen in Figure 7 , the original clause order read did not particularly influence the subjects' ability to switch styles. As can be seen in Figure 8, the subjects' recall for consistent descriptors decreased when they switched from the marked to the unmarked style. For tense, the opposite pattern was shown. As can be seen in Figure 9, the subjects' recall for consistent tense was impaired when the subjects switched from the unmarked to the marked form.

There were no style-read or serial-position effects for clause order and descriptor. For tense, the main effects for style read and serial position could not be calculated. There were no other significant interactions, except for a style-read $\times$ serial-position interaction for descriptor, caused by the overall characteristics produced decreasing for the marked-unmarked group, as compared with the unmarked-marked group.
Plot ratings were scored for Stories 1, 4, and 5. Averaging over the two plots, the plot averages for the plot-same groups were $2.68(S D=0.44), 2.99(S D=0.41)$, and 2.96 ( $S D=0.45)$, and the averages for the plot-change groups were $2.60(S D=0.49), 2.96(S D=0.48)$, and $2.82(S D=$ 0.42 ). It is difficult to examine plot ratings in more detail, because the two plots are not opposing and so cannot be scored in the same manner as the style characteristics.

\section{Discussion}

As can be seen from the Story 1 and Story 4 analyses, the subjects were able to learn the consistent style characteristics over the four stories. Unlike Experiment 1, a main effect for serial position was found for both descriptor and tense. This effect appears to be due to the increased power from the large number of subjects, as compared with Experiment 1. In addition, unlike the results of Experiment 1, a main effect for style read was found for descriptor. This was replicated in a combined analysis of all three experiments, indicating that this was due to in-

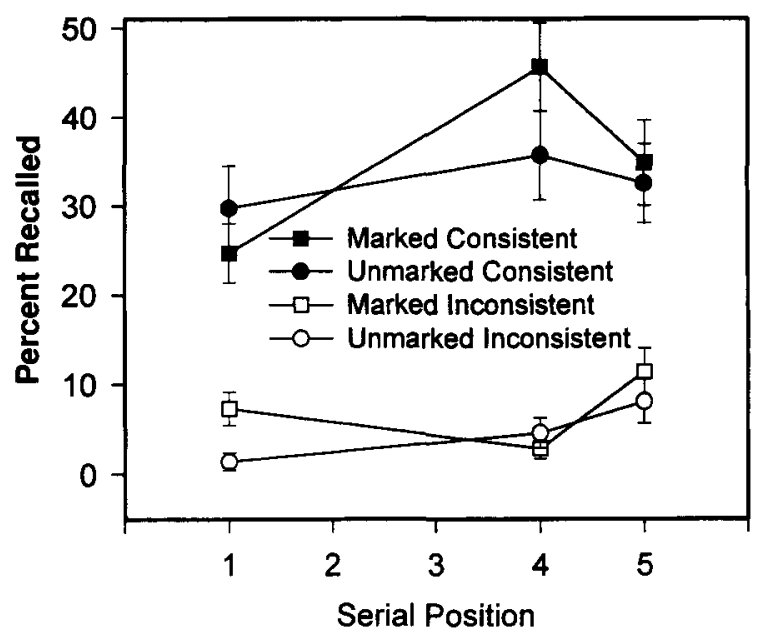

Figure 7. Percent of sentences recalled with consistent and inconsistent clause orders in Experiment 3 for switch-style groups, where marked is dependent-independent clause order and unmarked is independent-dependent clause order. Error bars are standard errors. 


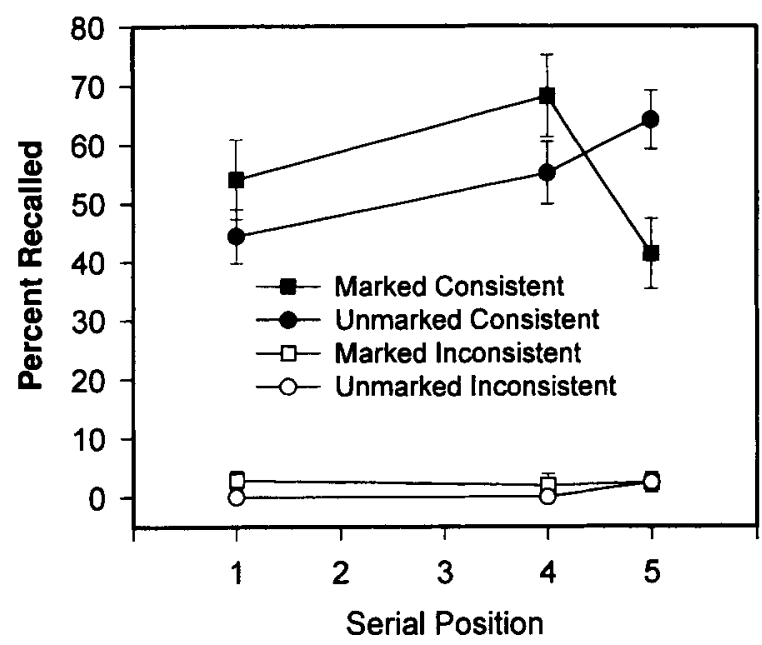

Figure 8. Percent of consistent and inconsistent descriptor types recalled in Experiment 3 for switch-style groups, where marked is phrase descriptors and unmarked is double descriptors. Error bars are standard errors.

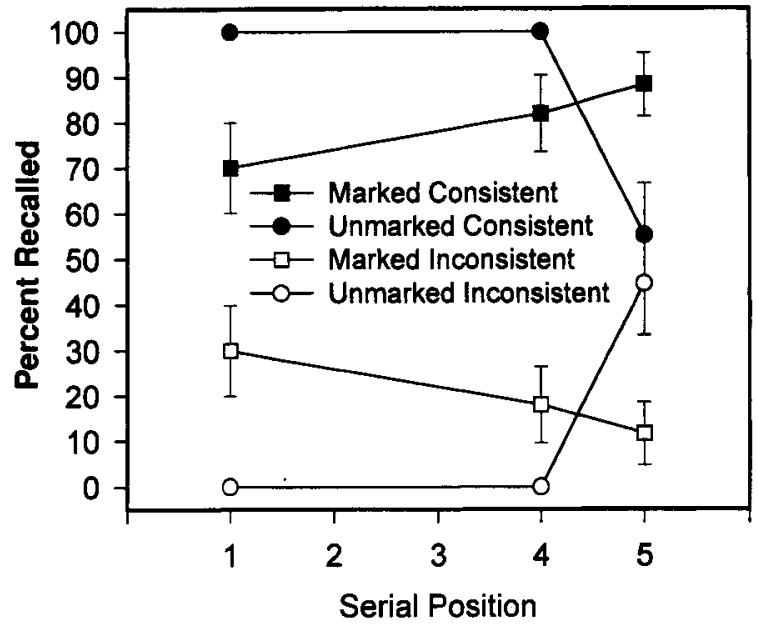

Figure 9. Percent of sentences recalled per serial position with consistent and inconsistent tense, for switch-style groups, where marked is present tense and unmarked is past tense. Error bars are standard errors.

creased power. Recall for marked and unmarked forms was generally equivalent by the fifth story for both Experiment 1 and Experiment 3.

Subjects were generally able to switch plot or style when these changed in the fifth story, although a significant consistency-of-style-produced $\times$ serial-position interaction suggests that switching impairs recall. The ability to switch depended on both the style characteristic considered and the qualities of markedness/unmarkedness. For clause order, all groups improved from Story 1 to Story 4 , and there appeared to be no particular differ- ences in switching. For descriptors, the subjects showed greater recall of style characteristics when switching from the unmarked to the marked descriptor form than vice versa. Since switching was aided by a increase in distinctiveness, this suggests that the descriptor learning was mostly explicit. In contrast, for tense, the subjects recalled the unmarked form better than the marked form in Stories 1 and 4 and showed difficulty in switching from the unmarked to the marked form in the fifth recall. This is consistent with there not being an easily accessible, explicit knowledge of tense.

The subjects' recall for plot was high and comparable with recall in Experiments 1 and 2. One difficulty in analyzing the plot data in more detail is that baseline for the other plot is not expected to be zero. Unlike the style characteristics, the two plots have a number of similarities in common and are not completely opposing.

\section{GENERAL DISCUSSION}

In Experiment 1, the subjects learned the regularities of both the style and the plot over successive recalls. This is the first demonstration of style learning using an arbitrary novel style. It shows that a style based on syntactic factors can be learned quickly, even under laboratory conditions. Previously, it could be argued that subjects were simply recognizing an established style and applying it to their recalls, rather than learning the particular characteristics of the material. Some of the style characteristics showed a high rate of learning over the first one or two recalls: tense was learned almost instantaneously. Others showed a slower learning pattern: clause order recall was initially low but doubled from the first to the fifth recall.

In Experiment 2, one group generated a new story after learning the style, and the other group listed the rules that applied to the learned stories. In the generate-story group, the subjects generated the more unnatural, or marked, forms to a higher degree than they did the more natural forms, for descriptors and clause order. Tense showed the opposite pattern; although perfect for the unmarked form, the subjects were poorer at generating the marked present tense in the new story. In the list-rules group, the subjects demonstrated a trend toward mentioning more rules for the marked forms than for the unmarked forms for all three style characteristics. Over all, there was an association, rather than a dissociation, between performance and listed rules. Tense was an exception to this, in that generate-story and list-rules performances showed opposite patterns. It is difficult to believe that subjects would show no knowledge of tense if extensively tested, and, as such, the list-rules results are consistent with, but not conclusive of, implicit learning.

In Experiment 3, the subjects learned four stories of the same plot and style and then read and recalled a fifth story that was the same or changed in plot, style, or both. When style was switched, the subjects were able to switch to the opposite form, but with some interesting 
Table 6

\begin{tabular}{|c|c|c|c|c|c|c|c|}
\hline \multirow[b]{3}{*}{ Effect or Interaction } & \multirow[b]{3}{*}{$d f$} & \multicolumn{6}{|c|}{ Characteristics } \\
\hline & & \multicolumn{2}{|c|}{ Clause } & \multicolumn{2}{|c|}{ Descriptor } & \multicolumn{2}{|c|}{ Tense } \\
\hline & & $F$ & $M S_{\mathrm{e}}$ & $F$ & $\overline{M S_{\mathrm{e}}}$ & $F$ & $M S_{\mathrm{e}}$ \\
\hline Consistency of style produced & 1,37 & 87.94 & 0.04 & 252.75 & 0.05 & 69.73 & 0.22 \\
\hline Consistency $\times$ serial position & 1,37 & 5.79 & 0.03 & 3.54 & 0.03 & 5.40 & 0.27 \\
\hline Style read $\times$ serial position & 1,37 & n.s. & n.s & 13.23 & 0.03 & n.c. & n.c. \\
\hline Style read $\times$ consistency $\times$ serial position & 1,37 & n.s. & n.s & 9,36 & 0.03 & 9.57 & 0.27 \\
\hline
\end{tabular}

Note-For descriptor, consistency $\times$ serial-position interaction, $p=.07$. For tense, style-read $\times$ serialposition interaction could not be calculated (n.c.), because $M S_{\mathrm{e}}=0$. n.s. $=$ not significant.

exceptions. Descriptor type and tense, although both highly learned in story recalls, showed opposite patterns. For descriptors, the subjects found it harder to switch from the marked to the unmarked form, whereas, for tense, the subjects found switching from the unmarked to the marked form more difficult. That is, the subjects showed a short-term fluency in imitating the correct tense in recall but, when required to switch, relied on normal production biases. Both Experiment 2 and Experiment 3 tense results are consistent with the classic implicit-learning finding of difficulty in transfer to related tasks.

Two approaches were explored in this study: a distinctiveness approach and an implicit-/explicit-learning approach. Stylistic text is considered inherently more distinctive than normal text. It is often more unusual or complex. These qualities allow material-appropriate processing for style, in that they slow comprehension and increase processing of surface information. Here, markedness was used as a definition of distinctiveness for optional forms. Distinctiveness can also be seen as defining an implicit/ explicit dimension. The more distinctive a characteristic is, the more likely it is to be noted explicitly and remembered. Recent work on implicit versus explicit learning has attempted more specific definitions of implicitness, such as a subjective threshold (Dienes \& Berry, 1997) or intentionality (Stadler, 1997), though no consensus yet exists. Although none of the work presented here meets such strict criteria of implicit learning, many of our findings can be interpreted in terms of the degree to which it is easy to explicitly note and later state a regularity. In this spirit, the implicit-/explicit-learning approach was used to shed light on our findings.

With the exception of tense, there was a positive relationship between rules stated and rules used in performance: the more verbalizable style characteristics were learned quickly and to a higher degree. Clause order did show a more gradual pattern of learning and was not as verbalizable as plot and descriptor type. The clause order results can be related to Green and Shanks's (1993) replication of a Hayes and Broadbent (1988) computer control task. Green and Shanks attributed the differences in performance for the implicit program, as compared with the explicit program (less recovery from a change in the underlying equation, lower performance under dual task conditions, lower asymptote level), to differences in difficulty level rather than to differences in implicitness/ explicitness. Although Green and Shanks's explanation fits the clause order results, it does not hold here for tense; tense was the easiest characteristic to learn but was the least verbalizable.

Implicit-learning studies have found a relation between salience and verbalizable knowledge. When the underlying rules to be deduced are made more salient, such as by the timing of events in a computer program (Berry $\&$ Broadbent, 1988) or by organization of the study strings (Reber, Kassin, Lewis, \& Cantor, 1980), performance improved and was made more verbalizable. Here, differences in the levels and patterns of learning were due to differences in the characteristics of the material. This is consistent with Whittlesea and Dorken's (1997) more parsimonious position that the learning found in implicitlearning tasks may be better understood by examining task demands and the structure of the stimuli.

A closer examination of the learning of style in text is long overdue. The presence of stylistic features in text is not irrelevant but has psychological consequences. Even in normal text, surface features are used to guide comprehension-for example, by drawing attention to particular qualities of words, by marking new topics, and by emphasizing parallel ideas. Since style is a quality that influences the readers' processing of text, it should be no surprise that style can influence the learning and production of text as well.

\section{REFERENCES}

ARISTotLE (1976). Poetics (I. Bywater, Trans.). In J. D. Kaplan (Ed.), The pocket Aristotle (12th ed., p. 345). New York: Washington Square Press.

BARTLETT, F. C. (1932). Remembering: A study in experimental and social psychology. London: Cambridge University Press.

BEGG, I. (1971). Recognition memory for sentence meaning and wording. Journal of Verbal Learning \& Verbal Behavior, 10, 176-181.

Berry, D. C., \& Broadbent, D. E. (1988). Interactive tasks and the implicit-explicit distinction. British Journal of Psychology, 79, 251272.

BoCK, K., Loebell, H., \& Morey, R. (1992). From conceptual roles to structural relations: Bridging the syntactic cleft. Psychological Review, 99, 150-171.

Bransford, J. D., Barclay, J. R., \& Franks, J. J. (1972). Sentence memory: A constructive versus interpretive approach. Cognitive Psychology, 3, 193-209.

BRansford, J. D., \& Franks, J. J. (1971). The abstraction of linguistic ideas. Cognitive Psychology, 2, 331-350.

BREWER, W. F., \& HAY, A. E. (1984). Reconstructive recall of linguistic style. Journal of Verbal Learning \& Verbal Behavior, 23, 237-249. 
Chapman, R. (1973). Linguistics and literature: An introduction to literary stylistics. London: Arnold.

D'ArCaIs, G. B. (1974). Is there a memory for sentences? Acta Psychologica, 38, 33-58.

DiENES, Z., \& BERRY, D. C. (1997). Implicit learning: Below the subjective threshold. Psychonomic Bulletin \& Review, 4, 3-23.

DienEs, Z., BRoADBENT, D. E., \& BeRRY, D. C. (1991). Implicit and explicit knowledge bases in artificial grammar learning. Journal of Experimental Psychology: Learning, Memory, \& Cognition, 17, 875887.

Enkvist, N. (1973). Linguistic stylistics. The Hague: Mouton.

FRANKS, J. J., \& BRANSFORD, J. D. (1971). Abstraction of visual patterns. Journal of Experimental Psychology, 90, 65-74.

FreEman, D. C. (1970). Linguistic approaches to literature. In D. C. Freeman (Ed.), Linguistics and literary style (pp. 3-17). New York: Holt, Rinehart \& Winston.

Goodman, G. O., McClelland, J. L., \& GibBs, R. W. (1981). The role of syntactic context in word recognition. Memory \& Cognition, 9 , $580-586$.

Gorrell, R. M., \& LaIRD, C. (1972). Modern English handbook (5th ed.). Englewood Cliffs, NJ: Prentice-Hall.

Graesser, A., \& MANDler, G. (1975). Recognition memory for the meaning and surface structure of sentences. Journal of Experimental Psychology: Human Learning \& Memory, 104, 238-249.

Green, R. E. A., \& Shanks, D. R. (1993). On the existence of independent explicit and implicit learning systems: An examination of some evidence. Memory \& Cognition, 21, 304-317.

HaRTLey, J., \& Homa, D. (1981). Abstraction of stylistic concepts. Journal of Experimental Psychology: Human Learning \& Memory, 7, 33-46.

Hayes, N. A., \& Broadbent, D. E. (1988). Two modes of learning for interactive tasks. Cognition, 28, 249-276.

Hyman, I. E., \& Rubin, D. C. (1990). Memorabeatlia: A naturalistic study of long-term memory. Memory \& Cognition, 18, 205-214.

IsocraTes (1934). (J. W. C. Atkins, Trans.). In Literary criticism in antiquity (Vol. 1, p. 128). Cambridge: Cambridge University Press.

Jou, J., \& HaRrIS, R. J. (1990). Event order versus syntactic structure in recall of adverbial complex sentences. Journal of Psycholinguistic Research, 19, 21-42.

Katz, S., \& Gruenwald, P. (1974). The abstraction of linguistic ideas in "meaningless" sentences. Memory \& Cognition, 2, 737-741.

Kelly, M. H., BocK, J. K., \& KeIL, F. C. (1986). Prototypicality in a linguistic context: Effects on sentence structure. Journal of Memory \& Language, 25, 59-74

KINTSCH, W., \& VAN DiJK, T. A. (1978). Toward a model of text comprehension and production. Psychological Review, 85, 363-394.

Lewicki, P., CZyzewska, M., \& Hoffman, H. (1987). Unconscious acquisition of complex procedural knowledge. Journal of Experimental Psychology: Learning, Memory, \& Cognition, 13, 523-530.

LORD, A. P. (1960). The singer of tales. Cambridge, MA: Harvard University Press.

MCDaniel, M. A. (1981). Syntactic complexity and elaborative processing. Memory \& Cognition, 9, 487-495.
Miall, D. S., \& Kuiken, D. (1994). Foregrounding, defamiliarization, and affect: Response to literary stories. Poetics, 22, 389-407.

MiLLER, G. A. (1962). Some psychological studies of grammar. American Psychologist, 17, 748-762.

Morris, C. D., Bransford, J. D., \& FranKs, J. F. (1977). Levels of processing versus transfer appropriate processing. Journal of Verbal Learning \& Verbal Behavior, 16, 519-533.

Mukarovsky, J. (1970). Standard language and poetic language. In D. C. Freeman (Ed.), Linguistics and literary style (pp. 40-56). New York: Holt, Rinehart \& Winston.

NoICE, H. (1991). The role of explanations and plan recognition in the learning of theatrical scripts. Cognitive Science, 15, 425-460.

OLSEN, D. R., \& FilBY, N. (1972). On the comprehension of active and passive sentences. Cognitive Psychology, 3, 361-381.

Plunkett, K., \& Marchman, V. (1993). From rote learning to system building: Acquiring verb morphology in children and connectionist nets. Cognition, 48, 21-69.

Posner, M. I., \& Keele, S. W. (1968). On the genesis of abstract ideas. Journal of Experimental Psychology, 77, 353-363.

Reber, A. S., Kassin, S. M., Lewis, S., \& Cantor, G. (1980). On the relationship between implicit and explicit modes in the learning of a complex rule structure. Journal of Experimental Psychology: Human Learning \& Memory, 6, 492-502.

REBER, A. S., \& LEWIS, S. (1977). Implicit learning: An analysis of the form and structure of a body of tacit knowledge. Cognition, $\mathbf{5}$, 333-361.

Reingold, E. M., \& MerikLe, P. M. (1988). Using direct and indirect measures to study perception without awareness. Perception \& Psychophysics, 44, 563-575.

RuBIN, D. C. (1977). Very long-term memory for prose and verse. Journal of Verbal Learning \& Verbal Behavior, 16, 611-621.

RUBIN, D. C. (1995). Memory in oral traditions. New York: Oxford University Press.

Rubin, D. C., \& WaLlaCe, W. T. (1989). Rhyme and reason: Analyses of dual retrieval cues. Journal of Experimental Psychology: Learning, Memory, \& Cognition, 15, 698-709.

Rubin, D. C., Wallace, W. T., \& Houston, B. C. (1993). The beginnings of expertise for ballads. Cognitive Science, 17, 435-462.

SACHS, J. S. (1967). Recognition memory for syntactic and semantic aspects of connected discourse. Perception \& Psychophysics, 2, 437-442.

SChmidt, J. A., McLaughin, J. P., \& Leighten, P. (1989). Novice strategies for understanding paintings. Applied Cognitive Psychology, 3, 65-72.

SchmidT, S. R. (1991). Can we have a distinctive theory of memory? Memory \& Cognition, 19, 523-542.

Shanks, D. R., \& ST. John, M. F. (1994). Characteristics of dissociable human learning systems. Behavioral \& Brain Sciences, 17, 367-447.

STADLER, M. A. (1997). Distinguishing implicit and explicit learning. Psychonomic Bulletin \& Review, 4, 56-62.

THORNDYKE, P. W. (1977). Cognitive structures in comprehension and memory of narrative discourse. Cognitive Psychology, 9, 77-110.

Whittlesea, B. W. A., \& Dorken, M. D. (1997). Implicit learning: Indirect, not unconscious. Psychonomic Bulletin \& Review, 4, 63-67. YouNG, J. N. (1961). 101 plots used and abused. Boston: The Writer.

\title{
APPENDIX \\ Sample Stories of the Two Plots and Two Styles
}

\author{
Style 1, Plot "Just Desserts" \\ The Cup of Coffee
}

In a small town it is a sweltering humid day. Because she dotes on him to the point of distraction, Dan Henderson, a married man, plans to murder his bland simple wife, Jane. When he takes her on a fishing trip they drive to a thickly wooded part of Maine. After they rise the next morning they eat a scrambled-egg breakfast together. When Jane's back is turned he drops fast acting poison into her coffee. As Jane seats herself Dan plans a tropical beach vacation. When Dan sees his fishing line move he runs to the nearby rippling stream. After Dan checks the empty line he returns to his woven wicker seat. As they drain their cups Jane smiles and says, "I know how you like your pitch black coffee hot." "Since my cup came out of the pot after yours I gave you my freshly made cup," Jane says. 


\section{APPENDIX (Continued)}

Style 2, Plot “Just Desserts"

The Killer

It was a steamy as a pressure cooker day in a small town. Dan Henderson, a married man, planned to murder his bland as toast wife, Jane, because she doted on him to the point of distraction. They drove to a part of Maine, isolated as Alaska, when he took her on a fishing trip. They ate a breakfast early as dawn together after they rose the next morning. Dan dropped poison, deadly as sin, into her coffee when her back was turned. Dan planned a vacation, remote as Antarctica, for himself as she seated herself. Dan ran to the near as a stone's throw stream when he saw his fishing line move. He returned to his woven like a basket seat after he checked the empty line. Jane smiled and said she knew how he liked his strong as the devil coffee hot, as they drained their cups. She said she gave him her fresh as a newborn cup since her's came out the pot after his.

\section{Style 1, Plot "Switch" \\ The Bag}

At an Arizona shopping mall it is a desert dry day. As she walks by the counter Pat, a shoplifter, hides stolen goods in her white paper bag. While a security guard looks on, Pat walks towards the revolving glass doors. That he has seen a shoplifter with a white bag, the guard informs the slightly balding manager. So that he can cut her off, the manager strides towards the revolving door exits. When Pat sees the exit is covered, Pat walks into a pink painted room for changing. While a woman is busy changing, Pat sidles up to the lady's quilted suede purse. After she switches bags, Pat walks out with the tiny suede purse. While she waves Pat's bag the lady runs after brunette-haired Pat. While Pat walks through the store doors the manager detains the disheveled-clothed lady.

\section{Style 2, Plot "Switch"}

The Shoplifter

It was a dry as the desert day at an Arizona shopping mall. Pat, a shoplifter, hid stolen goods in her large as an elephant bag as she walked by the counters. Pat walked towards the revolving like a carousel doors while a security guard looked on. The guard told the bald as a billiard ball manager that he had seen a shoplifter with a white bag. The manager strode towards the close as a shout exits so that he could cut her off. Pat walked into a pink as a petunia changing room when she saw the exit was covered. Pat sidled up the a lady's quilted like a blanket purse while the woman was busy changing. Pat walked out with the cute as a button purse after she switched bags. The lady ran out after Pat, fleeing like an escaping con, while she waved Pat's bag. The manager detained the lady, disheveled as a bag lady, while Pat walked through the store doors.

(Manuscript received January 21, 1997;

revision accepted for publication June $9,1997$. 